\title{
Long-term effects of porcine zonae pellucidae immunocontraception on ovarian function in feral horses (Equus caballus)
}

\author{
J. F. Kirkpatrick ${ }^{1}$, I. M. K. Liu ${ }^{2}$, J. W. Turner, Jr ${ }^{3}$, R. Naugle ${ }^{4}$ and \\ R. Keiper ${ }^{4}$
}

'Deaconess Research Institute, $252017 \mathrm{th}$ St, W., Billings, MT 59102, USA; ${ }^{2}$ Department of Reproduction, University of California, Davis CA 95616, USA; ${ }^{3}$ Department of Physiology, Medical College of Ohio, Toledo, OH 43699, USA; and ${ }^{4}$ Department of Biology, Pennsylvania State University, Mont Alto, PA 17237, USA

\begin{abstract}
Summary. Ten feral mares free-roaming in Maryland, USA, were inoculated with porcine zonae pellucidae (PZP) protein before the breeding season for three consecutive years (1988-90). Ovarian function was monitored for 51 days during the peak of the breeding season after the third annual PZP inoculation, in seven of these mares and in four untreated control mares, by means of urinary oestrone conjugates and nonspecific progesterone metabolites. None of the ten inoculated mares became pregnant in 1990 , compared with $55 \%$ of 20 control mares, which included two of the four monitored for ovarian function. Three of the untreated mares demonstrated apparent normal ovarian activity, characterized by preovulatory oestrogen peaks, concurrent progesterone nadirs at ovulation, breeding activity, and luteal-phase progesterone increases after ovulation. Two of the seven monitored PZP-treated mares demonstrated ovulatory cycles that did not result in conception. One was pregnant as a result of conception in 1989 and demonstrated a normal, late-gestation, endocrine profile. The remaining four PZP-treated mares revealed no evidence of ovulation, and urinary oestrogen concentrations were significantly depressed. The experiments indicated that (i) a third consecutive annual PZP booster inoculation is $>90 \%$ effective in preventing pregnancies in mares and (ii) three consecutive years of PZP treatment may interfere with normal ovarian function as shown by markedly depressed oestrogen secretion.
\end{abstract}

Keywords: zona pellucida; contraception; ovary; immunocontraception: oestrogen; progestin; horse

\section{Introduction}

Immunization with porcine zonae pellucidae (PZP) is a promising approach to immunocontraception. This glycoprotein layer is composed of several protein fractions of which a fraction of $M_{\mathrm{r}} 55000$, referred to as ZP3, has been identified in the species studied to be the specific zona receptor for sperm recognition and attachment (Sacco et al., 1984).

Immunization with PZP has resulted in significant zonae antibody titres and fertility inhibition in a range of laboratory species (Tsunoda \& Chang, 1976; Gwatkin et al., 1977) and non-human primates (Gulyas et al., 1983; Sacco et al., 1983a, 1987; Aitken et al., 1984; Bamezai et al., 1986; Dunbar et al., 1989). Sacco (1977), Hasegawa et al. (1985) and Trounson et al. (1980) have demonstrated that human and porcine zonae share common antigens. In several of these studies, the administration of PZP or purified ZP3 was accompanied by disruption of ovarian function and 
cyclic endocrine patterns, but, in most cases, normal ovarian function returned over an interval ranging from several cycles to 300 days.

A concern regarding the potential use of PZP, ZP3 or any antigenic epitope of ZP3 in humans or exotic species is the possible permanent loss of fertility or the disruption of normal reproductive endocrine events. Studies in three species have indicated adverse side-effects on ovarian function. Mahi-Brown et al. (1985) found that immunization of bitches led to long-term alterations in oestrous cycles and to abnormal steroid profiles. Immunization of rabbits with PZP also led to disruption of hormone profiles (Wood et al., 1981; Skinner et al., 1983). Ten baboons immunized with ZP3 showed reduced oestrogen concentrations in plasma and numbers of antral follicles; two of these animals failed to ovulate, and five became amenorrheic by 8 months after immunization (Dunbar et al., 1989).

Liu et al. (1989) demonstrated that PZP immunization could render mares infertile, but that fertility returned during the following breeding season. Kirkpatrick et al. (1990a) also used PZP to inhibit fertility in free-roaming feral horses and demonstrated that fertility returned during the next breeding season. In a continuation of that study, Kirkpatrick et al. (1991) demonstrated that a single annual booster inoculation of PZP was sufficient to extend the contraceptive efficacy of PZP through a second breeding season.

Despite the data accumulated from studies of non-human primates and horses, little is known about the effects of continuous PZP treatment on the mammalian ovary over several years. The present study evaluated the effectiveness of monitoring ovarian function in uncaptured feral mares by means of urinary steroid metabolites and determined the effects of PZP immunization for three consecutive years upon fertility, ovarian endocrine function, and sexual behaviour in feral mares.

\section{Materials and Methods}

\section{Animals}

Ten free-roaming feral mares inhabiting Assateague Island National Seashore, MD, USA, were evaluated for the contraceptive efficacy of a third consecutive annual PZP inoculation; ovarian function was assessed in seven of these mares. Twenty untreated mares served as controls and ovarian function was studied in four of them. Ages and fertility records were known for six of the seven PZP-immunized mares and for three of the four control mares. Ages ranged from 3 to 16 years. Each mare possessed unique markings and was photographed for later identification.

\section{PZP immunization}

The PZP antigen was prepared from porcine ovaries as described by Liu et al. (1989) and stored frozen until used in the field. In 1988 (year 1 of this study), the ten experimental mares were immunized with two or three inoculations of PZP. The schedule of inoculations and the resulting contraceptive effects have been described (Kirkpatrick et al.. 1990a).

In February, 1989 (year 2 of this study) the ten PZP-treated mares were given a single PZP booster inoculation and the contraceptive efficacy was assessed (Kirkpatrick et al., 1991). The ten mares were given a further single booster inoculation in March 1990 (year 3 of this study) identical to the booster treatment in year 2 . Between 4 May and 24 June 1990, urine samples in soil or sand were collected daily or every other day, without capture, from seven of these ten treated mares and four untreated control mares. The selection of the seven mares from among the ten was made on the basis of accessibility for urine collections, and the timing of the collections was based on peak breeding activity among the Assateague population (Keiper \& Houpt, 1984). The age distributions of the 20 mares selected as controls for the assessment of contraceptive efficacy was similar to that of the ten treated mares. Pregnancy was determined during October 1990, on the basis of concentrations of urinary oestrone conjugates as described by Kirkpatrick $e t a l$. (1988). Differences in rates of pregnancy between treated and control mares were tested for significance by means of binomial probability distribution (Freedman et al., 1978).

\section{Behavioural assessments}

Sexual behaviour of treated and control mares was assessed in terms of behavioural oestrous patterns including (i) raised tail when stallions attempted mounting, (ii) eversion of the clitoris, (iii) absence of kicking or biting by the mare during attempted or successful mountings and (iv) copulation, as described by Waring (1983). The period for which 
each mare was observed each day ranged from $\mathrm{I}$ to $3 \mathrm{~h}$ depending upon the duration of the interval between sighting and urination.

\section{Assessment of ovarian function}

Unextracted urine samples, collected from the soil, were assayed for oestrone conjugates and non-specific progesterone metabolites by microtitre enzymeimmunoassays. The oestrone conjugate assay has been described (Daels et al., 1991) and validated for the Equidae (Evans et al., 1984; Czekala et al., 1990; Monfort et al., 1991) and reflects plasma oestrogen concentrations (Daels et al., 1991); the inter- and intra-assay coefficients of variation were $11 \cdot 10(n=7)$ and $3.82 \%(n=7)$, respectively. The lower limit of sensitivity for the assay was $6.25 \mathrm{pg}$ and concentrations of oestrone conjugates in serially diluted urine samples extracted from soil have exhibited parallelism to the standard curve (Kirkpatrick et al., 1988). Recovery of ${ }^{3} \mathrm{H}$-labelled steroid metabolites from the soil has been demonstrated to be $>95 \%$ (Kirkpatrick et al., 1988).

The enzymeimmunoassay for non-specific progesterone metabolites has been described and validated in the Equidae by Kirkpatrick et al. (1990b) and employs monoclonal antibody 1284-1 raised against $20 \delta$-hydroxyprogesterone (provided by $R$. Chatterton. Northwestern University). The inter- and intra-assay coefficients of variation were $12.38(n=8)$ and $10.42 \%(n=8)$, respectively. The lower limit of sensitivity was $0.075 \mathrm{ng}$. To account for differences in urine concentrations, all urine samples were assayed for creatinine by the microcolorimetric method of Taussky (1954), and steroid metabolite concentrations were converted to $\mathrm{ng} / \mathrm{mg}$ creatinine. Differences in mean hormone concentrations were tested for significance by means of Student's $t$ test.

\section{Results}

None of the ten mares treated with PZP for the third consecutive year became pregnant by October 1990 , but 11 of 20 control mares (55\%) were pregnant in October 1990. The difference in pregnancy rates was significant $(P<0 \cdot 001)$. Two of the four control mares for which breeding-season serial urine samples were collected were pregnant in October 1990.

The four control mares monitored for ovarian function demonstrated evidence of pregnancy and parturition (M13, 15 years), oestrous cycles without conception (Speckles, age unknown but $<10$ years, and M4, 18 years) and a conceptive cycle (M17GL, 3 years). Pregnancy and parturition (Fig. 1a) was characterized by concentrations of urinary oestrone conjugates which fell rapidly $\sim 7$ days before parturition and urinary non-specific progesterone metabolite concentrations which fell abruptly on the day before parturition. There were no endocrine indications of post-partum ovulation during the 39 days of urine collection following parturition. A marked increase in oestrone conjugates occurred 6 days after parturition, but, on the basis of non-specific progesterone metabolite concentrations, did not culminate in ovulation; this increase in oestrone conjugates was accompanied by repeated behavioural oestrus in the presence of the band stallion. A second control mare demonstrated an ovulatory oestrous cycle (Fig. 1b) based on urinary oestrone conjugates and non-specific progesterone metabolites, with a clear peak in preovulatory oestrone conjugates ( $375 \mathrm{ng} / \mathrm{mg}$ creatinine) coinciding with a non-specific progesterone metabolite nadir ( $20 \mathrm{ng} / \mathrm{mg}$ creatinine) and a subsequent luteal-phase increase in non-specific progesterone metabolites (330 ng/mg creatinine). Urinary non-specific progesterone metabolites reached basal concentrations ( $15 \mathrm{ng} / \mathrm{mg}$ creatinine) by Day 36 of collection, without behavioural or endocrine evidence for a second oestrous cycle. At the same time as the preovulatory increase in oestrone conjugates, this mare demonstrated behavioural oestrus in the presence of the band stallion. A third control mare demonstrated a conceptive ovulatory oestrus (Fig. 1c) characterized by an exceptionally high peak in preovulatory oestrone conjugates $(1 \cdot 1 \mu \mathrm{g} / \mathrm{mg}$ creatinine) coinciding with a nadir in nonspecific progesterone metabolite ( $27 \mathrm{ng} / \mathrm{mg}$ creatinine) and a subsequent and sustained rise in this urinary metabolite $(1.7 \mu \mathrm{g} / \mathrm{mg}$ creatinine $)$. Concentrations of non-specific progesterone metabolites remained above $1.0 \mu \mathrm{g} / \mathrm{mg}$ creatinine throughout the remainder of the collection period. Urinary oestrone conjugates fell to basal concentrations $(15-55 \mathrm{ng} / \mathrm{mg}$ creatinine) for 35 days after the ovulatory nadir in non-specific progesterone metabolites and then rose abruptly to $1.45 \mu \mathrm{g} / \mathrm{mg}$ creatinine, which is a characteristic signal of pregnancy in mares (Evans et al., 1984). During the first 5 days of urine collection, which coincided with the preovulatory oestrone conjugate increases, 
M17GL displayed behaviour associated with oestrus and was witnessed being successfully mounted on Days 3-5 of collection. By October, M 17GL had a urinary oestrone conjugate concentration of $31.3 \mathrm{ng} / \mathrm{mg}$ creatinine, indicating that the pregnancy had terminated, a common event in feral 3-year-old mares (Lucas et al., 1990). The fourth control mare produced a foal on 6 April, 1 month before the initiation of urine collection. Urinary oestrone conjugates and non-specific progesterone metabolite concentrations revealed a portion of an ovulatory cycle during the first 20 days of the collection period and a second ovulatory cycle during the 31 remaining days of the collection period.

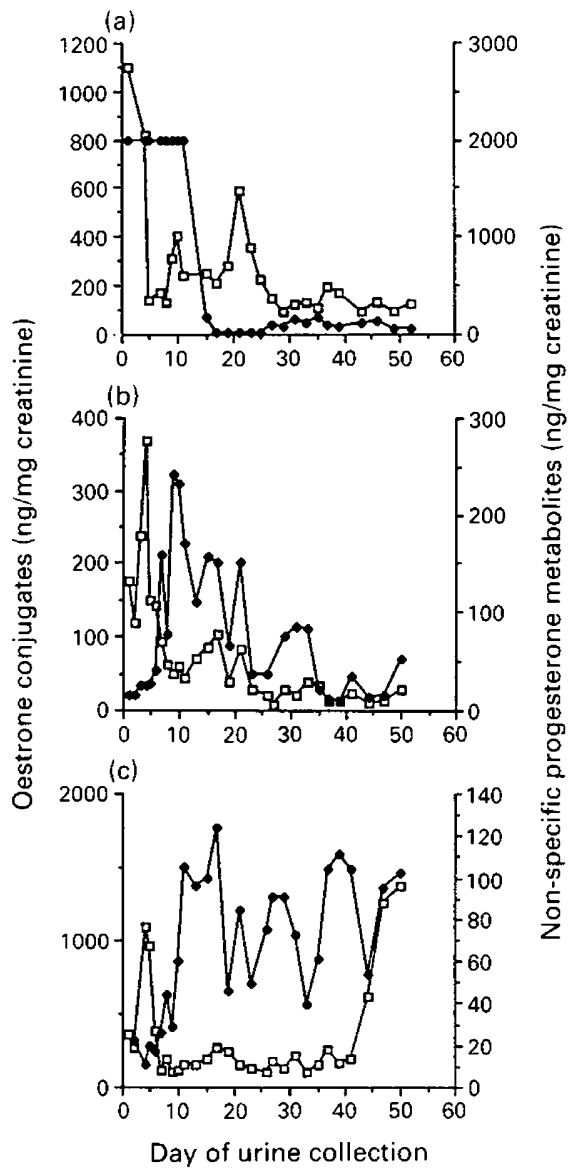

Fig. 1. Profiles of urinary oestrone conjugates $(\square)$ and progesterone metabolites $(\diamond)$ in three control mares: (a) M13 (a foal was born on Day 12), (b) an oestrous cycle without conception in Speckles and (c) an oestrous cycle with conception in M17GL. Day 0 was 4 May.

Four of the seven PZP-treated mares (T5AE, 10 years; M17GJ, 5 years; M2E, 10 years; M13F's Bay, age unknown) displayed no endocrine evidence of ovulation during the 51-day collection period. Concentrations of urinary non-specific progesterone metabolites revealed no luteal-phase increases. Similarly, urinary oestrone conjugate concentrations and patterns revealed no signs of an oestrous cycle and concentrations during the 51-day collection period were extremely variable and significantly lower $(P<0.05)$ than those of the control mares Speckles and M4, which demonstrated ovulatory cycles. Neither T5AE nor M17GJ displayed any signs of behavioural oestrus during the study. M2E displayed three incidences of behavioural oestrus on Days 16, 20 and 35 of 
collection, in the absence of any significant increases in oestrone conjugates, but the band stallion showed no interest in this mare during these displays. The mare M13F's Bay had a significant increase in oestrone conjugates between Days 25 and 35 of collection, which was accompanied by eversion of the clitoris and four successful mounts by the band stallion during this time. Urinary endocrine profiles are shown for two of these non-cyclic mares in Fig. 2a, b.

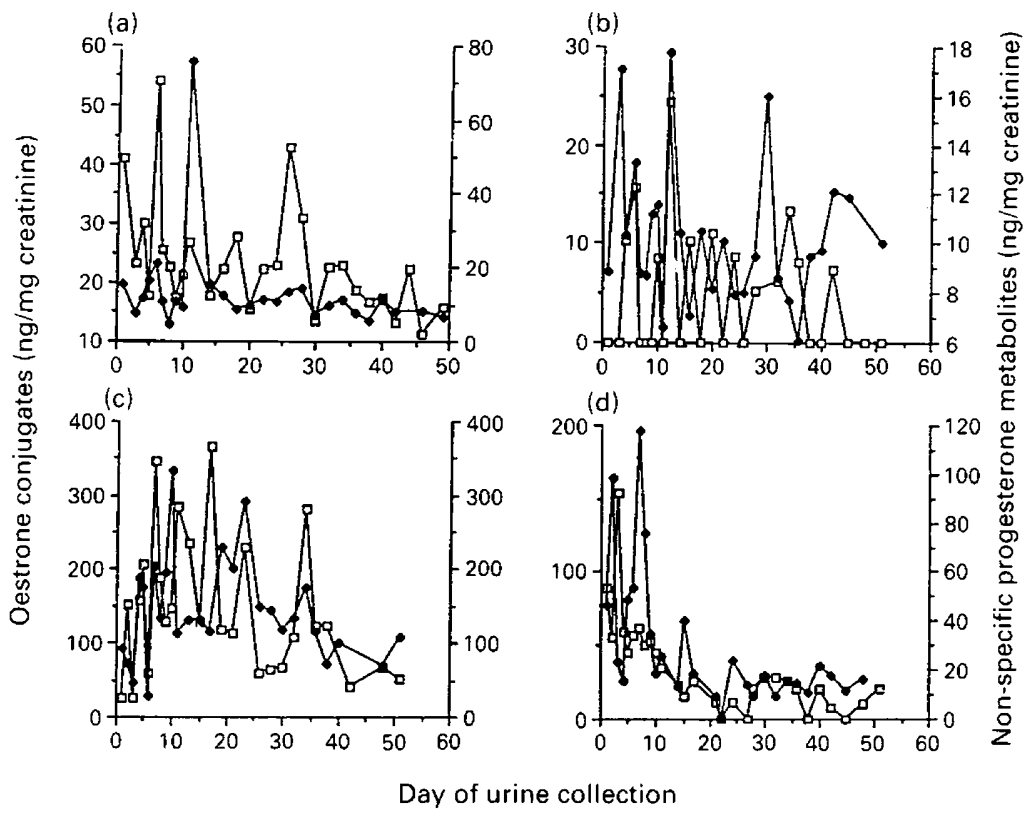

Fig. 2. Profiles of urinary oestrone conjugates $(\square)$ and progesterone metabolites $(\bullet)$ in four mares treated with porcine zonae pellucidae: non-ovulatory patterns of urinary steroids in (a) T5AE and (b) M2E; ovulatory cycles without conception in (c) M6I and (d) T2BE.

The PZP-treated mares M6I (6 years old) and T2BE (10 years old) displayed urinary nonspecific progesterone concentrations and patterns characteristic of ovulatory oestrous cycles (Fig. 2c, d, respectively), but mare M6I did not have the characteristic preovulatory peak in oestrone conjugates.

During two of the increases in oestrone conjugates, on the 10th and 34th days of collection, M6I displayed tail raising and evoked attention from the band stallion, but no mounting was observed. Urine collection with T2BE began in the latter stages of the luteal phase, concentrations of nonspecific progesterone metabolites falling from $199 \mathrm{ng} / \mathrm{mg}$ creatinine to non-detectable concentrations 15 days later. T5AE displayed intermittent behavioural oestrus throughout most of the collection period despite very low oestrone concentrations of conjugates and on one occasion, on Day 27 of collection, was mounted successfully by the band stallion; this mounting coincided with a rapid rise in concentrations of oestrone conjugates from non-detectable to $45 \mathrm{ng} / \mathrm{mg}$ creatinine.

The seventh PZP-treated mare (M17G, 8 years old) was pregnant at the time of urine collection and produced a foal on 1 August 1990. This was the only foal produced among the mares treated with a booster inoculation in the second year of this study. The date of parturition indicates an unusually late conception ( $\sim 25$ August) for Assateague mares and suggests that her antibody titres had fallen to a concentration which allowed conception. Urinary endocrine data for oestrone conjugates and non-specific progesterone metabolites were consistent with data on plasma hormones for mares in late pregnancy (Ginther, 1979). 


\section{Discussion}

This study demonstrates that a single annual PZP booster inoculation given after 2 years of treatment will extend the observed infertility in such treated mares for a third year. Only two of the seven monitored PZP-treated mares demonstrated ovulatory cycles and it is probable that treatment with PZP for 3 years altered ovarian function. The absence of a luteal phase in four of these mares, coupled with significantly lower and intermittent increases in oestrone conjugates may indicate incomplete folliculogenesis, with reduced secretion of oestrogen and failure to ovulate. These alterations in ovarian function are consistent with data from PZP-treated rabbits (Skinner et al., 1983, 1984), dogs (Mahi-Brown et al., 1985) and baboons (Dunbar et al., 1989).

The urinary endocrine data and the correlated behavioural observations and events, such as parturition and breeding behaviour for the four control mares, reflect functional ovaries. These data provide an initial view of ovarian function in feral mares which is consistent with data derived from domestic horses based on plasma steroid concentrations (Ginther, 1979) and urinary steroid metabolites (Daels et al., 1991).

Three previous studies with horses point to long-term treatment, as opposed to a single treatment, as the factor which resulted in the alteration of ovarian function in PZP-treated mares. Liu et al. (1989) treated mares with PZP for only one breeding season and demonstrated the occurrence of ovulation after 7 months of infertility. This was indicated by normal patterns of luteal-phase plasma progesterone and normal fertility rates for up to 3 years after the single inoculation. These results are consistent with the proposed mechanisms of contraceptive action of antibodies to PZP in mares, which include a fertilization block brought about by steric hindrance of the sperm receptor (Liu et al., 1989) and an acrosome reaction in horse spermatozoa induced by zona pellucida (Arns et al., 1990). Kirkpatrick et al. (1990a, 1991) demonstrated the return of normal fertility rates among feral mares 1-2 years after a single immunization with PZP and, although ovarian endocrine function was not monitored in these two studies, the normal fertility rates indicate that ovulation occurred and that preovulatory oestrogen concentrations were sufficient to elicit breeding behaviour. Similarly, short-term active immunization of non-human primates led to infertility, but with undetectable or only temporary disruption of ovarian function (Gulyas et al., 1983; Sacco et al., 1983a, b, 1987; Bamezai et al., 1986).

Zonae antibodies have been shown to bind to the developing follicular zona pellucida as well as to the zona of ovulated ova (Henderson et al., 1988). This may indicate that zonae antibodies disrupt some developmentally critical cellular communication between differentiating follicular cells and the growing oocyte (Skinner et al., 1990), which ultimately leads to the destruction of a portion or all of the oocyte pool (Skinner et al., 1984).

At least three factors may play a significant role in long-term alterations of ovarian function. The duration of contraceptive efficacy is directly related to the amount of PZP antigen administered (Henderson et al., 1988) and to the resulting antibody titres (Millar et al., 1989), and ovarian dysfunction may likewise be related to PZP dose.

The purity of the PZP antigen may be a second factor in the possible alteration of ovarian function. Most of the early studies with rabbits, dogs and some non-human primates which demonstrated ovarian dysfunction used crude preparations of PZP, which included the entire complement of zonae protein. These various preparations also include associated carbohydrate moieties which are thought to be important antigenic components (Paterson \& Aitken, 1990). More recent studies have used preparations of higher purity and even genetically engineered epitopes of the ZP3 protein. Dunbar et al. (1989) used both ZP1 and ZP3, which share a common antigenic epitope, but serious ovarian dysfunction still occurred in baboons after 49 weeks and nine cycles. The horses in this study received crude PZP antigen which may have exacerbated long-term effects despite the small dose of $65 \mu \mathrm{g}$.

The duration of treatment may contribute to long-term effects. Small doses of high purity may produce long-term effects through cumulative effects upon follicular pools. No other mammals, 
apart from the horses in this study, have been treated with PZP for 3 years. Thus, our current understanding of long-term effects is poor. Based on the work of Liu et al. (1989) with captive mares, and the initial field tests with feral mares (Kirkpatrick et al., 1990a, 1991), all of which used the same antigen and doses and demonstrated a return to normal fertility, the data from three consecutive years of treatment suggest that the duration of treatment may be the primary factor for this observed ovarian dysfunction in mares.

The authors gratefully thank G. Olson, Assateague Island National Seashore, for logistical support, B. L. Lasley, UC-Davis, for the oestrone conjugate antibodies, and A. G. Sacco, Wayne State University, for the careful review of the manuscript, and $\mathrm{K}$. Asis for assistance in collecting urine samples. This study was funded by the World Society for the Protection of Animals and National Park Service Grant CA-1600-30005.

\section{References}

Aitken, R.J., Richardson, D.W. \& Hulme, M. (1984) Immunological interference with the properties of the zona pellucida. In Immunological Aspects of Reproduction in Mammals, pp. 305-325. Ed. D. B. Crighton. Butterworths, London.

Arns, M.J., Webb, G.W., Johnson, L., Martin, J., Welsh, T. \& Evans, J.W. (1991) Zona pellucida-induced acrosome reactions in equine spermatozoa. $J$. Reprod. Fert. Suppl. 44, 738-739.

Bamezai, A.K., Suman, D.C. \& Talwar, G.P. (1986) Effect of immunization against pig zona pellucida (PZP) on steroid hormone profiles and fertility in primates. J. Reprod. Immunol. 9 (Suppl.), 85.

Czekala, N.M., Kasman, L.H., Allen, J., Oosterhuis, J. \& Lasley, B.L. (1990) Urinary steroid evaluations to monitor ovarian function in exotic ungulates. VI. Pregnancy detection in exotic Equidae. Zoo Biol. 9, 4348.

Daels, P.F., Ammon, D.C., Stabenfeldt, G.H., Liu, I.M.K., Hughes, J.P. \& Lasley, B.L. (1991) Urinary and plasma estrogen conjugates, estradiol and estrone concentrations in nonpregnant and early pregnant mares. Theriogenology 35, 1001-1015.

Dunbar, B.S., Lo, C., Powell, J. \& Stevens, V.C. (1989) Use of a synthetic peptide adjuvant for the immunization of baboons with denatured and deglycosylated pig zona pellucida glycoproteins. Fert. Steril. 52, $311-318$.

Evans, K.L., Hughes, J.P., Couto, M., Kasman, L.H. \& Lasley, B.L. (1984) Pregnancy diagnosis in the domestic horse through direct urinary estrone conjugate analysis. Theriogenology 22, 615-620.

Freedman, D., Pisani, B. \& Purres, R. (1978) Statistics. W.W. Norton, NY.

Ginther, O.J. (1979) Reproductive Biology of the Mare: Basic and Applied Aspects. McNaughton \& Gunn, Ann Arbor, MI.

Gulyas, B.J., Gwatkin, R.B.L. \& Yuan, L.C. (1983) Active immunization of cynomolgus monkeys (Macaca fascicularis) with porcine zona pellucida. Gamete Res. 4, 299-307.

Gwatkin, R.B.L., Williams, D.T. \& Carlo, D.J. (1977) Immunization of mice with heat solubilized hamster zonae: production of anti-zona antibody and inhibition of fertility. Fert. Steril. 28, 871-877.
Hasegawa, A., Ikeda, Y., Li, Y., Shigeta, M., Koyama, K. \& Isojima, S. (1985) Blocking effect of antiserum raised to purified porcine zona pellucida antigen on sperm binding to oocytes in humans. Acta Obstet. Gynaec. Jap. 37, 2639a.

Henderson, C.J., Hulme, M.J. \& Aitken, R.J. (1988) Contraceptive potential of antibodies to the zona pellucida. J. Reprod. Fert. 83, 325343.

Keiper, R. \& Houpt, K. (1984) Reproduction in feral horses: an eight-year study. Am. J. Vet. Res. 45, 991-995.

Kirkpatrick, J.F., Kasman, L.H., Lasley, B.L. \& Turner, J.W. (1988) Pregnancy diagnosis in uncaptured feral horses. J. Wildl. Mgmt 52, 305-308.

Kirkpatrick, J.F., Liu, I.K.M. \& Turner, J.W. (1990a) Remotely-delivered immunocontraception in feral horses. Wildl. Soc. Bull. 18, 326-330.

Kirkpatrick, J.F., Lasley, B.L. \& Shideler, S.E. (1990b) Urinary steroid evaluations to monitor ovarian function in exotic ungulates. VII. Urinary progesterone metabolites in the Equidae assessed by immunoassay. Zoo Biol. 9, 341-348.

Kirkpatrick, J.F., Liu, I.K.M., Turner, J.W. \& Bernoco, M. (1991) Antigen recognition in feral mares previously immunized with porcine zonae pellucidae. $J$. Reprod. Fert. Suppl. 44, 321-325.

Liu, I.K.M., Bernoco, M. \& Feldman, M. (1989) Contraception in mares heteroimmunized with pig zonae pellucidae. J. Reprod. Fert. 85, 19-29.

Lucas, Z., Raeside, J.I. \& Betteridge, K.J. (1991) Noninvasive assessment of the incidences of pregnancy and pregnancy loss in the feral horses of Sable Island. J. Reprod. Fert. Suppl. 44, 146-147.

Mahi-Brown, C.A., Yanagimachi, R., Hoffmann, J.C. \& Huang, T.T.F. (1985) Fertility control in the bitch by active immunization with porcine zona pellucida: use of different adjuvants and patterns of estradiol and progesterone levels in estrous cycles. Biol. Reprod. 32, 761-772.

Millar, S.E., Chamow, S.M., Baur, A.W., Oliver, C., Robey, F. \& Dean, J. (1989) Vaccination with a synthetic zona pellucida peptide produces long-term contraception in female mice. Science, NY 246, 935-938.

Monfort, S.L., Arthur, N.P. \& Wildt, D.E. (1991) Monitoring ovarian function and pregnancy by evaluating 
excretion of urinary oestrogen conjugates in semifree-ranging Przewalski's horses (Equus przewalskii). J. Reprod. Fert. 91, 155-164.

Paterson, M. \& Aitken, R.J. (1990) Development of vaccines targeting the zona pellucida. Curr. Opin. Immunol. 2, 743-747.

Sacco, A.G. (1977) Antigenic cross-reactivity between human and pig zona pellucida. Biol. Reprod. 16, $164-173$.

Sacco, A.G., Subramanian, M.G., Yurewicz, E.C., DeMayo, F.J. \& Dukelow, W.R. (1983a) Heteroimmunization of squirrel monkeys (Saimiri sciureus) with a purified porcine zonae antigen (PPZA): immune response and biological activity of antiserum. Fert. Steril. 39, 350-358.

Sacco, A.G., Yurewicz, E.C. \& Zhang, S. (1983b) Immunoelectrophoretic analysis of porcine zonae pellucidae. J. Reprod. Fert. 68, 21-31.

Sacco, A.G., Subramanian, M.G. \& Yurewicz, E.C. (1984) Association of sperm receptor activity with a purified pig zona antigen (PPZA). J. Immunol. 6, 89-103.

Sacco, A.G., Pierce, D.L., Subramanian, M.G., Yurewicz, E.C. \& Dukelow, W.R. (1987) Ovaries remain functional in squirrel monkeys (Saimiri sciureus) immunized with porcine zona pellucida 55,000 macro-molecule. Biol. Reprod. 36, 48 I-490.

Skinner, S.M., Kirchick, H.J. \& Dunbar, B.S. (1983) Abnormal cellular differentiation of ovarian follicles is induced by immunization with zona pellucida (ZP). J. Cell Biol. 97, 182a.

Skinner, S.M., Mills, T., Kirchick, H.J. \& Dunbar, B.S. (1984) Immunization with zona pellucida proteins results in abnormal ovarian follicular differentiation and inhibition of gonadotropin-induced steroid secretion. Endocrinology 115, 2418-2432.

Skinner, S.M., Timmon, T.M., Schwoebel, E.D. \& Dunbar, B.S. (1990) The role of zona pellucida antigens in fertility and infertility. Immun. Allergy Clin. N.A. 10, 185-197.

Taussky, H.H. (1954) A microcolorimetric determination of creatinine in urine by the Jaffe reaction. $J$. Biol. Chem. 208, 853-861.

Trounson, A.O., Shivers, C.A., McMaster, R. \& Lopata, A. (1980) Inhibition of sperm binding and fertilization of human ova by antibody to porcine zona pellucida and human sera. Arch. Androl. 4, 29-35.

Tsunoda, Y. \& Chang, M.C. (1976) Effect of antirat ovary antiserum on the fertilization of the mouse and hamster eggs in vivo and in vitro. Biol. Reprod. 14, $354-361$.

Waring, G.H. (1983) Horse Behavior. Noyes Publications, Park Ridge, NJ.

Wood, D.M., Liu, C. \& Dunbar, B.S. (1981) Effect of alloimmunization and heteroimmunization with zona pellucida on fertility in rabbits. Biol. Reprod. 25, 439-450.

Received 18 January 1991 